\title{
Health Literacy among Older Adults during COVID-19 Pandemic: A Cross-Sectional Study in an Urban Community in Thailand
}

\author{
Kirada Pechrapa ${ }^{1}$, Korravarn Yodmai ${ }^{1}$, Wirin Kittipichai ${ }^{1}$, Phithaya Charupoonpol ${ }^{1}$, Wanich Suksatan ${ }^{2}$ \\ ${ }^{1}$ Department of Family Health, Faculty of Public Health, Mahidol University, Bangkok, Thailand \\ ${ }^{2}$ Faculty of Nursing, HRH Princess Chulabhorn College of Medical Science, Chulabhorn Royal Academy, Bangkok, Thailand
}

Corresponding Author:

Korravarn Yodmai, $\mathrm{PhD}, \mathrm{RN}$

Department of Family Health, Faculty

of Public Health, Mahidol University,

Bangkok 73170, Thailand

E-mail: korravarn.yod@mahidol.edu

ORCID:

https://orcid.org/0000-0002-7608-5552

Received: August 18, 2021

Revised: October 23, 2021

Accepted: October 28, 2021
Background: This study assessed health literacy $(\mathrm{HL})$ and its associated factors among older adults during the coronavirus disease 2019 (COVID-19) pandemic. Methods: This cross-sectional study included older adults in urban communities in Thailand. We randomly selected a total of 421 older adults and performed multiple logistic regression analyses. Results: The average age of the respondents was $70.0 \pm 7.1$ years. Most respondents were female, married, had a low education level (primary school or lower), were unemployed, and had sufficient income. We observed nonproficient and proficient HL levels in $56.1 \%$ and $43.9 \%$ of respondents, respectively. The factors influencing $\mathrm{HL}$ included attending healthcare services at a tertiary hospital, good accessibility to COVID-19 prevention materials and health information, and provision of a high level of social support from neighbors and health personnel. Conclusion: The results of our study provide important information on the outcome of accessibility and social support among older adults in an urban community during the COVID-19 pandemic. In addition, access to health services, prevention equipment, and information was important for people at risk of $\mathrm{HL}$ and helped promote good behaviors.

Key Words: Health literacy, COVID-19, Frail eldery, Health promotion

\section{INTRODUCTION}

The coronavirus disease 2019 (COVID-19) global pandemic began at the end of 2019. ${ }^{1,2)}$ Within 1 year, 208 million individuals worldwide were infected by this virus and 4.38 million individuals died. ${ }^{3)}$ In Thailand, 948,442 cases of COVID-19 have been confirmed, with 730,437 cured and 7,973 deaths. ${ }^{3}$ The pandemic affected all age groups, causing economic deterioration and ensuing health problems. ${ }^{4)}$ More than $50 \%$ of fatalities occurred among older adults owing to increased complications and longer lengths of hospital admission compared to those in other populations. ${ }^{5)}$ As a result, treatment costs and numbers of lost family members were high. ${ }^{6}$ COVID-19 prevention behaviors were extremely important in reducing viral spread and casualties in populations at risk. ${ }^{7)}$

As COVID-19 is transmitted by droplets, ${ }^{8)}$ health authorities in several countries, including Thailand, promoted frequent hand washing with soap and water, use of masks, and social distancing to reduce exposure, in addition to strict citywide lockdowns to control the spreads of the epidemic. ${ }^{9)}$ Moreover, accessing fundamental health information, obtaining a genuine understanding of the principles of disease prevention, and managing public health remained crucial, especially for at-risk populations. ${ }^{10,11)}$ Making decisions about how to follow health advice was one of the processes necessary for COVID-19 prevention behaviors. ${ }^{12)}$

Health literacy (HL) is a concept addressed in healthcare systems and research on health promotion. ${ }^{13)}$ It constitutes the process by which individuals acquire knowledge and motivation and how they understand and access information, express opinions, and make decisions about promoting and maintaining health. ${ }^{14,15)}$ A recent study applied HL to promote health among chronically ill 
patients. ${ }^{16,17)}$ Previously, older adults with poor education or limited access to health information (AHI) received health information from healthcare workers and community health volunteers. ${ }^{18,19)}$ Because physical deterioration affects the ability to learn new knowledge, HL regarding health problems faced by older adults have gradually been learned over longer periods compared to other population groups. Related studies have demonstrated inadequate HL among older adults, especially those with cognitive impairment and poor vision and hearing. ${ }^{19)}$ Individuals with low education levels also have low HL scores and tend to have worse communication skills and lack critical HL. ${ }^{18,19)}$ However, healthcare communication is extremely important for maintaining and promoting health and well-being.

In the context of Thai communities, families are characterized by nuclear and extended families, meaning that family members, relatives, and neighbors can provide interaction and support regarding the physical and psychological well-being of older adults. ${ }^{20)}$ However, during the COVID-19 pandemic, relatives, neighbors, and public health workers were educated and built an understanding of protection measures using mass media, including online networks, to provide information designed for older adults. ${ }^{21)} \mathrm{HL}$ on managing common health problems differs from that in critical or epidemic situations as it constitutes a new epidemic, in which unclear knowledge related to COVID-19 prevention behaviors and HL were lacking among older adults. ${ }^{18)}$

A literature review on HL in the COVID-19 context revealed that few studies have assessed factors associated with $\mathrm{HL}$ among older adults in Thailand. Studies on the general public, university students, and factors related to the COVID-19 pandemic have all been conducted. ${ }^{22,23)}$ However, during COVID-19, older adults are in triple jeopardy, especially if they reside in crowded areas, such as urban communities. Furthermore, although many older adults were advised to self- quarantine and avoid direct contact with those who may infect them, their social connections in the community were one of the most important supportive resources available.

As mentioned above, the prevention of COVID-19 among older adults in crisis areas such as urban communities required their increased HL in addition to efforts to assess and explore factors influencing $\mathrm{HL}$ in this population. This information can be used for developing and implementing interventions and policies. When developing the conceptual framework for the present study, we applied the ecological assessment of the PRECEDE-PROCEED model ${ }^{24)}$ to determine the predisposing, reinforcing, and enabling factors that influenced HL among older adults in an urban community. The model explained the predisposing factor as the basis on which individuals increased their motivation to perform a behavior, the reinforcing factor as that which encouraged individuals to perform a particular behavior, and enabling factor as the environmental influencing factor that directly affected the behavior to support or prevent the degradation of behavior among individuals. This study assessed and determined factors influencing $\mathrm{HL}$ among older adults living in urban communities during the pandemic.

\section{MATERIALS AND METHODS}

\section{Research Design and Sampling}

This cross-sectional community-based survey was conducted in Ubon Ratchathani Province, Thailand, which has the highest proportion of older adults at a risk of COVID-19. The selection criteria of the subjects were male and female sex, age 60 years and older, residence in the study area over 2 years, and ability to participate while collecting data. We excluded older adults who could not speak the Thai language and were unable to communicate or diagnose cognitive impairments.

We applied a multistage sample technique. First, one district in each district health zone was chosen for sampling from among the three district health zones in Ubon Ratchathani Province, Thailand. Second, in each district, one urban community, also known as a municipal area, was chosen. Finally, we selected a target population at random using a community name list from four districts in Northeast Thailand. We calculated the sample size using $G^{*}$ Power. ${ }^{25)}$ The effect size was 0.30 , with an alpha level of 0.05 and a power of 0.80 . The resulting calculated sample size was 384 . To prevent data loss, an additional $10 \%$ of subjects were included. Thus, this study included a total of 421 subjects. Based on sample size, we randomly selected 105-106 individuals from each urban community.

The protocol for this study was reviewed and approved by the Ethics Review Committee (IRB) of the Faculty of Public Health, Mahidol University (MUPH 2020-156). An informed consent statement was obtained before commencement of the data collecting.

\section{Research Instrument}

Data were collected using self-reported questionnaires that were conducted in the respondents' homes and communities. A panel of three experts rated the overall content validity of all instruments at 1.00 and the index of item objective congruence was over 0.5. We assessed the interrater reliability of the self-reported questionnaire between the principal and co-principal investigators in all settings in a pilot test of 30 participants, which showed acceptable values of $0.71-0.93$, with structured questionnaires comprising the 
six parts described below.

(1) Access to health services (AHS) was developed based on literature reviews. AHS included two questions. First, where the respondents accessed their healthcare services. The healthcare services in the study area comprised a subdistrict health-promoting hospital (primary healthcare unit), a community hospital, and a regional hospital (tertiary hospital). Second, the assessment of the ease of accessing healthcare services involved three individual items: "was the hospital far from your home?," "was the cost of travel expensive," and "were you able to pay the extra treatment costs?" The Cronbach's alpha was 0.77.

(2) Access to COVID-19 preventive materials was developed based on literature reviews. It comprised five items: access to alcohol-based hand rub, access to free alcohol-based hand rub or soap, access to masks, access to free masks, and access to face shields. Respondents who always had access to these preventive measures earned one point, with scores ranging from 0 to 5 points. Good access to COVID-19 preventive material was defined as individuals who were able to access all materials (five points). The Cronbach's alpha was 0.77 .

(3) AHI comprised nine items related to AHI from health personnel, health volunteers, neighbors, village headman, TV, radio, internet, family members, and documents. For example, one question asked, "did you regularly access health information from public health officials?" A positive response was scored one point. The scores ranged from 0 to 9 points. Good access health to information was defined as scores of 7-9. The Cronbach's alpha was 0.71.

(4) Social support was developed by applying the social support theory described by $\mathrm{Cobb},{ }^{26)}$ which comprises eight items. Each item involved four rating scales (never, rarely, sometimes, and always), and each was scored from 1 to 4 points. The total score ranged from 8 to 32 points. A high level of received social support from family members was defined as total scores of 25-32 points. The Cronbach's alpha was 0.79 .

Social support from neighbors was developed by applying the social support theory proposed by $\mathrm{Cobb}^{26)}$ and comprised seven items. Each item involved four rating scales (never, rarely, sometimes, and always), and each was scored from 1 to 4 points. The total score ranged from 7 to 28 points. A high level of social support was defined as a total score of 22-28. The Cronbach's alpha was 0.88 .

Social support from health personnel was developed similar to others. ${ }^{26)}$ It comprised seven items with four rating scales as described above. The total score ranged from 7 to 28 points. A high level of received social support was defined as a total score of $22-$ 28. The Cronbach's alpha was 0.93 .

(5) HL was developed by the Health Education Department of the Ministry of Public Health, Thailand. ${ }^{27)}$ It comprises 24 items in the following six elements: AHI, understanding, interaction to change, decision-making, modification, and discussion of health. The responses used a five-point Likert scale: strongly disagree $=1$, disagree $=2$, neither agree nor disagree $=3$, agree $=4$, and strongly agree $=5$. Total scores of $96-120$ points indicated proficient HL, in which individuals had adequate HL. The Cronbach's alpha was 0.93 .

(6) The sociodemographic variables of the study population consisted of four items with open-ended questions with multiple-choice responses, including age, sex, marital status, and current work.

\section{Statistical Analysis}

Data were analyzed using the Statistical Package for the Social Sciences version 18.0 (SPSS Inc., Chicago, IL, USA). Frequency, percentage, mean, and standard deviation were used to describe respondent characteristics. To explore factors associated with $\mathrm{HL}$, we performed multiple logistic regression analysis, with the results presented as odds ratios (ORs) and $95 \%$ confidence intervals (CIs). Statistical significance was set at $\mathrm{p}<0.05$.

\section{RESULTS}

\section{Characteristics of the Study Population}

The average age of the respondents was $70.0 \pm 7.1$ years; most respondents $(53.4 \%)$ were aged 60-69 years, followed by $70-79$ years $(36.1 \%)$ and 80 years and over (10.5\%). Overall, $62.7 \%$ of respondents were female and $37.3 \%$ were male. Over $60 \%$ of respondents were married, $30.4 \%$ were separated/divorced/widowed, and $5.5 \%$ were single. Almost $40 \%$ of respondents were employed, $43.5 \%$ were farmers, $28.5 \%$ were business owners, and $28.0 \%$ were temporary employees (Table 1 ).

\section{Study Variables}

The predisposing factors included education level, income sufficiency, and chronic illness. Most subjects had graduated at the primary school level or lower (63.4\%), possessed sufficient income (69.6\%), and experienced chronic illness (61.5\%). Among reinforcing factors, $83.4 \%, 76.9 \%$, and $70.1 \%$ of respondents received sufficiently high levels of social support from family members, neighbors, and health personnel, respectively.

Among enabling factors, most of the respondents accessed healthcare services at district (53.4\%), regional (23.8\%), and subdistrict health-promoting (22.8\%) hospitals. Moreover, $76.7 \%$ reported that receiving healthcare services in these hospitals was convenient. Regarding accessing COVID-19 prevention materials, 
Table 1. Factors associated with HL among older adults by binary logistic regression analysis

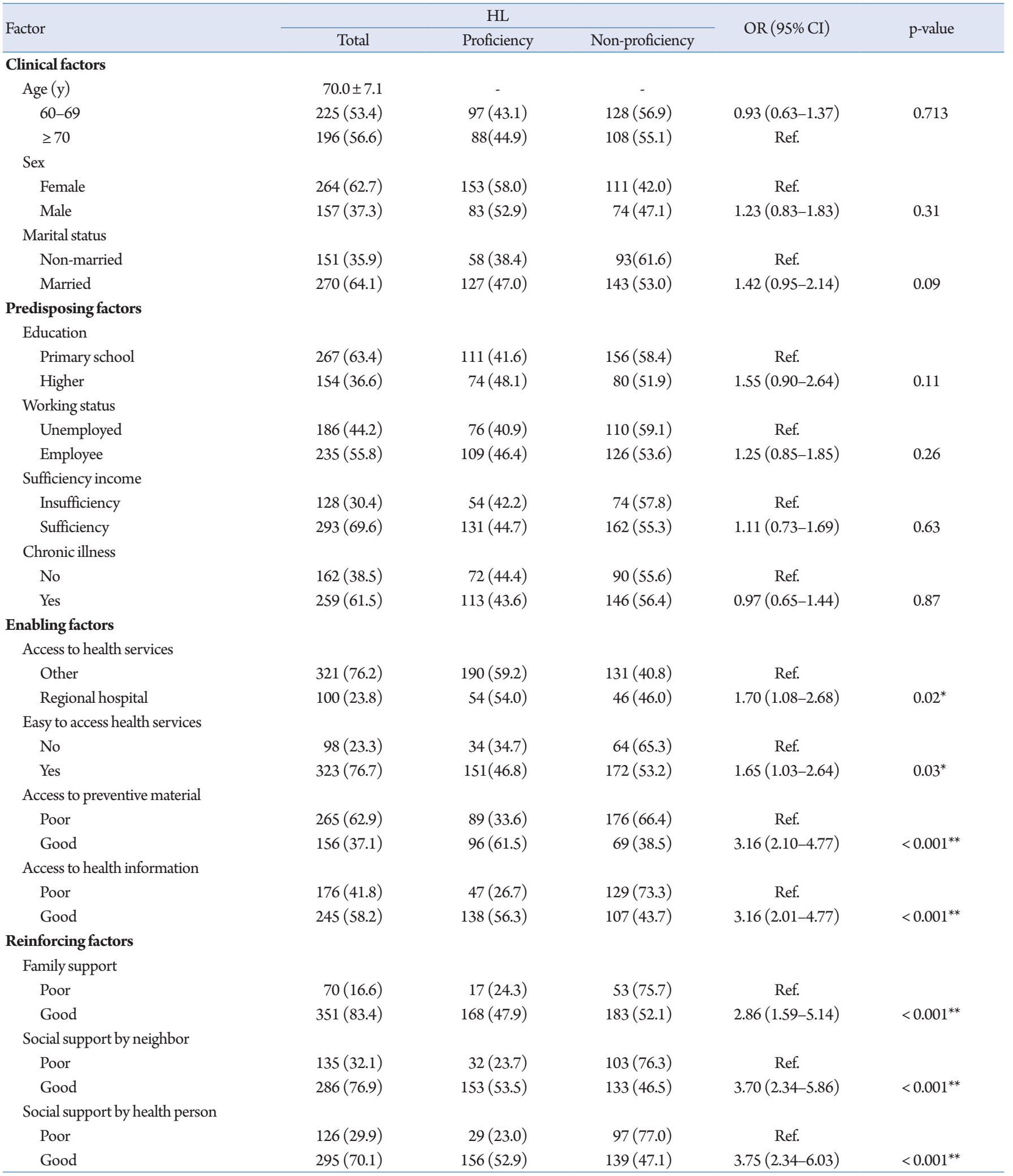

Values are presented as mean \pm standard deviation or number $(\%)$.

$\mathrm{HL}$, health literacy; OR, odds ratio; $\mathrm{CI}$, confidence interval.

${ }^{*} \mathrm{p}<0.05,{ }^{* *} \mathrm{p}<0.01$. 
most respondents $(62.9 \%)$ replied that they did not access them; however, $58.2 \%$ reported good AHI (Table 1 ).

\section{HL in the study population}

We observed nonproficient and proficient HL in $56.1 \%$ and $43.9 \%$ of Thai older adults, respectively, during the COVID-19 pandemic in this study.

\section{Factors associated with proficient $H L$ among older adults}

Table 1 indicates that no predisposing factors, such as education level, sufficient income, and chronic illness, were associated with proficient HL ( $p>0.05)$. Regarding enabling factors, AHS, convenient AHS, access to preventive materials, and AHI were associated with proficient HL. Our findings indicated that older adults accessing healthcare services at regional hospitals were 1.7 times more likely to have proficient $\mathrm{HL}(\mathrm{OR}=1.70$; 95\% CI, 1.08-2.68; $\mathrm{p}=0.02$ ). Respondents with convenient access to healthcare services were 1.65 times more likely to have proficient $\mathrm{HL}$ $(\mathrm{OR}=1.65 ; 95 \% \mathrm{CI}, 1.03-2.64 ; \mathrm{p}=0.03)$. Regarding access to preventive materials, participants who could access all materials were 3.16 times more likely to have proficient $\mathrm{HL}(\mathrm{OR}=3.16$; 95\% CI, 2.10-4.77; p < 0.001). Lastly, participants with high levels of AHI were 3.16 times more likely to have proficient $\mathrm{HL}$ $(\mathrm{OR}=3.16$; 95\% CI, 2.10-4.77; $\mathrm{p}<0.001)$ (Table 1).

Regarding reinforcing factors, older adults receiving high-level social support from family members were 2.86 times more likely to have proficient $\mathrm{HL}(\mathrm{OR}=2.86$; 95\% CI, 1.59-5.14; $\mathrm{p}<0.001)$. In addition, older adults receiving high levels of social support from neighbors were 3.7 times more likely to have proficient $\mathrm{HL}$ $(\mathrm{OR}=3.70$; 95\% CI, 2.34-5.86; $\mathrm{p}<0.001)$. Finally, those receiving high levels of social support from health personnel were 3.7 times more likely to have proficient $\mathrm{HL}(\mathrm{OR}=3.75 ; 95 \% \mathrm{CI}, 2.34-6.03$; $\mathrm{p}<0.001$ ) (Table 1).

Multiple logistic regression analysis of reinforcing factors showed that high levels of social support from neighbors and health personnel significantly predicted proficient $\mathrm{HL}$ in older adults. Participants receiving high levels of social support from their neighbors were more than twice as likely to have proficient $\mathrm{HL}$ compared to those receiving low levels of support $(\mathrm{OR}=2.02$; 95\% CI, 1.16-3.53; $\mathrm{p}<0.01$ ). Additionally, those who received high levels of social support from health personnel were more than twice as likely to have proficient HL compared to those who had low levels $(\mathrm{OR}=2.55$; 95\% CI, 1.47-4.41; $\mathrm{p}<0.01)$. However, while high levels of social support from family members were more likely to predict HL, HL did not differ from those receiving low levels of support $(p>0.05)$ (Table 2).

The enabling factors predicted significantly proficient HL $(\mathrm{p}<0.05)$. Older adults accessing health services at regional hospitals had adequate $\mathrm{HL}(\mathrm{OR}=2.53$; 95\% CI, 1.47-4.34; $\mathrm{p}<0.01)$, and older adults with convenient access to healthcare services were

Table 2. Factors associated with HL in older adults by multiple logistic regression analysis

\begin{tabular}{llr}
\hline Variable & OR $(95 \% \mathrm{CI})$ & $\mathrm{p}$-value \\
\hline Predisposing factors & & 0.358 \\
$\quad$ Young older adults $(<70 \mathrm{y})$ & $1.240(0.78-1.96)$ & 0.544 \\
Male & $1.158(0.72-1.86)$ & 0.052 \\
Married & $1.618(0.99-2.63)$ & 0.274 \\
Higher education & $1.321(0.80-2.17)$ & 0.961 \\
Current working & $0.988(0.62-1.59)$ & 0.365 \\
Sufficient income & $0.790(0.47-1.32)$ & 0.862 \\
Chronic illness & $1.042(0.66-1.65)$ & 0.709 \\
Reinforcing factors & & $<0.01^{* *}$ \\
Received high level of social support from family members & $1.150(0.56-2.36)$ & $<0.01^{* *}$ \\
Received high level of social support from neighbors & $2.030(1.15-3.59)$ & $<0.01^{* *}$ \\
Received high level of social support from health personnel & $2.860(1.62-5.06)$ & $0.02^{*}$ \\
Enabling factors & & $<0.01^{* *}$ \\
Accessed health services at regional hospital & $2.250(1.27-3.97)$ & $<0.01^{* *}$ \\
Convenient to access health services in hospitals & $1.890(1.09-3.29)$ & $1.920(1.18-3.12)$ \\
High level of access to health information & $2.760(1.72-4.41)$ & \\
Accessed COVID-19 prevention material & & \\
\hline
\end{tabular}

HL, health literacy; OR, odds ratio; CI, confidence interval; COVID-19, coronavirus disease 2019.

${ }^{*} \mathrm{p}<0.05,{ }^{* *} \mathrm{p}<0.01$. 
1.76 times more likely to have adequate HL compared to those without convenient AHS in hospitals ( $\mathrm{OR}=1.76$; $95 \% \mathrm{CI}$, 1.023.02; $\mathrm{p}=0.04$ ). Concerning access to materials for COVID-19 prevention, participants with good access were twice as likely to have adequate and proficient HL compared to those without such access $(\mathrm{OR}=2.62 ; 95 \% \mathrm{CI}, 1.66-4.13 ; \mathrm{p}<0.01)$. Participants with good AHI were 1.95 times more likely to have adequate HL compared to those without AHI (OR=1.95; 95\% CI, 1.21-3.13; $\mathrm{p}<0.01$ ) (Table 2).

\section{DISCUSSION}

Our study explored factors influencing HL among older adults. The results showed that their HL was proficient and associated with better accessibility and social support. Individuals with proficient $\mathrm{HL}$ can experience healthy lives free from infectious diseases. HL not only affects a person's awareness of disease prevention but also has a greater effect on the earlier management of health problems. Proficient HL predicted good preventive behaviors at rates almost twice those of the other groups. Gautam et al. ${ }^{28)}$ suggested that $\mathrm{HL}$ affected perceptions and behaviors related to individual protection from COVID-19 and followed the pharmacologic management of their health problems. A high proportion of $\mathrm{HL}$ is linked to prevention behaviors and low rates of COVID-19 nationwide.

The urgency for professionals to act and promote behavioral changes in the population is high during a pandemic. HL is a leading factor in improving awareness of risk issues and decision-making regarding changing health behaviors and lifestyles. ${ }^{17,29,30)}$ Proficient HL led to ideal health behaviors and a new normal lifestyle to reduce the incidence of COVID-19 transmission. ${ }^{9)}$ The older adults in urban communities in our study demonstrated adequate health knowledge and competencies very similar to those reported by related studies. ${ }^{18,19)}$ We observed non-proficient and proficient HL in $56.1 \%$ and $43.9 \%$ of respondents, respectively. Previously, over half of the older adults had insufficient HL or nonproficiency. The sample population's residence in urban or semiurban settings may have resulted in a better quality of life related to education level, living conditions, and access to information. ${ }^{31)}$ Although older adults in rural communities receive more support, many areas were locked down and activities canceled. ${ }^{32)}$ However, women were one of the most vulnerable populations during the pandemic and often had less access to information compared to men. ${ }^{33)}$ Therefore, their lifestyles may differ. ${ }^{31}$

Moreover, our study indicated that social support was a reinforcing factor for HL among older adults, similar to other studies. Social support from neighbors is important to older adults, particu- larly those in Asian countries. ${ }^{18,34,35)}$ Neighbors are also important for communicating health information. ${ }^{36)}$ Even when they are living with children, most individuals spend their free time with neighbors in the community. ${ }^{36)}$ Moreover, neighbors serve as role models of behavior change and can be useful in promoting health. ${ }^{37,38)}$ Thus, the HL of older adults is delivered through neighbors. ${ }^{39,40)}$ When a neighbor has good HL, the older adults will also have good $\mathrm{HL}^{40)}$ In addition, when the society is strong, vulnerable individuals will be protected and enjoy good health and well-being, leading to equal AHS. ${ }^{37}$

Unsurprisingly, we found that older adults supported by good health professionals also experienced good HL. In addition, AHS and health information were related to HL. This likely occurred because $\mathrm{HL}$ arises from health promotion by health personnel. ${ }^{18)}$ As mentioned above, the enabling factors include environmental factors that directly influence behavior and prevent illnesses stemming from such behaviors. ${ }^{37)}$ Therefore, the ability to access health services is a fundamental variable correlating to $\mathrm{HL}$ and health behavior. However, we found that the experience of accessing health services in tertiary hospitals was significantly associated with HL. Tertiary hospitals are large specialized hospitals providing health services that secondary and primary hospitals cannot. ${ }^{41)}$ They have both talent and expertise in providing healthcare services. Although the level of hospital expertise was related to health knowledge among older adults in this study, the number of subjects was small and insufficient to understand the process of establishing health knowledge. Additional studies are needed to explore health promotion and communication skills in tertiary hospitals to promote healthful decisions and healthcare. ${ }^{42)}$

However, we found that education levels, adequate income, and chronic illnesses such as hypertension, diabetes, and cardiovascular disease were not associated with HL in older adults. To explain this, most of the older adults in our study experienced chronic illness; they also know each other well in their community and participated and share health information, which may have influenced their HL and the stability of their health behaviors. Thus, older adults with chronic diseases showed no associations with $\mathrm{HL}$ in our study, contrary to the findings of related studies. ${ }^{29,43)}$ The literature on health knowledge arising from the learning process has demonstrated improved health knowledge following educational interventions in individuals with low education levels. ${ }^{26)}$ In contrast, while highly educated people better can learn health information and understand health, their HL is directly influenced by the academic level obtained. ${ }^{44)}$ Thus, our results differed from those of related studies. ${ }^{29,43)}$

Our study had several limitations. First, the sample was not statistically representative of older adults because we included only 
urban communities in Northeast Thailand. Future studies should compare HL in older adults in urban, suburban, and rural communities in several settings. Second, this was the first study to use the AHS, concerning access to COVID-19 preventive materials, AHI, social support, social support from neighbors, social support from health personnel, and HL questionnaires, which was on only one aspect of its validity (content validity). Future studies should use the whole aspect of validity (content and reliability) based on reliable and valid questionnaires. Finally, because of the cross-sectional design, this study could not determine causal relationships, but only associations and correlations. However, our results demonstrated that $\mathrm{HL}$ among older adults after the COVID-19 pandemic could limit its transmission and permit social activities necessary for the health of older adults. Indeed, we observed higher levels of HL among older adults who accessed health services at regional hospitals compared to the levels in those who accessed other healthcare facilities. Based on our results, further studies should focus on the spread of fake news or incorrect information (e.g., vaccination) that might be associated with HL, as well as the use of health service models aimed at HL among older adults. Policymakers should also consider ways to reduce gaps and disparities in health services in vulnerable populations.

In conclusion, the results of this study demonstrated high levels of $\mathrm{HL}$ among older adults residing in urban communities. We also found that AHS, easily accessed preventive material, and AHI predicted HL in older adults. Promoting proficient HL among older adults is important. Additionally, social support from family, neighbors, and health personnel was associated with HL in older adults. Strong social support can encourage high levels of HL and healthy behaviors in this population. Family, neighbors, and health personnel are important, and policymakers should consider effective channels of communication and health promotion. Lastly, good access to health care services, preventive material, and health information predicted high levels of HL. AHS, prevention equipment, and convenient information are important for people at risk of $\mathrm{HL}$ and to promote good behaviors.

\section{ACKNOWLEDGEMENTS}

We thank to the Department of Family Health, Faculty of Public Health, Mahidol University for their support.

\section{CONFLICT OF INTEREST}

The researchers claim no conflicts of interest.

\section{AUTHOR CONTRIBUTIONS}

Conceptualization, KP, KY, WK, PC, WS. Data curation, KP, KY, WS. Writing-original draft, KP, KY, WK, PC. Writing-review \& editing, KP, KY, WK, PC.

\section{REFERENCES}

1. Huang C, Wang Y, Li X, Ren L, Zhao J, Hu Y, et al. Clinical features of patients infected with 2019 novel coronavirus in Wuhan, China. Lancet 2020;395:497-506.

2. Zhu N, Zhang D, Wang W, Li X, Yang B, Song J, et al. A novel coronavirus from patients with pneumonia in China, 2019. N Engl J Med 2020;382:727-33.

3. Worldometer. COVID-19 Coronavirus pandemic: report of coronavirus cases [Internet]. [place unknown]: Worldometer; c2021 [cited 2021 Nov 6]. Available from: https://www.worldometers.info/coronavirus/.

4. Edwards E, Janney CA, Mancuso A, Rollings H, VanDenToorn A, DeYoung M, et al. Preparing for the behavioral health impact of COVID-19 in Michigan. Curr Psychiatry Rep 2020;22:88.

5. Shahid Z, Kalayanamitra R, McClafferty B, Kepko D, Ramgobin D, Patel R, et al. COVID-19 and older adults: what we know. J Am Geriatr Soc 2020;68:926-9.

6. LeRoy AS, Robles B, Kilpela LS, Garcini LM. Dying in the face of the COVID-19 pandemic: contextual considerations and clinical recommendations. Psychol Trauma 2020;12:S98-9.

7. Yodmai K, Pechrapa K, Kittipichai W, Charupoonpol P, Suksa$\tan \mathrm{W}$. Factors associated with good COVID-19 preventive behaviors among older adults in urban communities in Thailand.J Prim Care Community Health 2021;12:21501327211036251.

8. Armitage R, Nellums LB. COVID-19 and the consequences of isolating the elderly. Lancet Public Health 2020;5:e256.

9. Suksatan W, Choompunuch B, Koontalay A, Posai V, Abusafia AH. Predictors of health behaviors among undergraduate students during the COVID-19 pandemic: a cross-sectional predictive study.J Multidiscip Healthc 2021;14:727-34.

10. Choompunuch B, Suksatan W, Sonsroem J, Kutawan S, In-udom A. Stress, adversity quotient, and health behaviors of undergraduate students in a Thai university during COVID-19 outbreak. Belitung Nurs J 2021;7:1-7.

11. Sadang JM, Palompon DR, Suksatan W. Older adults' experiences and adaptation strategies during the midst of COVID-19 crisis: a qualitative instrumental case study. Ann Geriatr Med Res 2021;25:113-21.

12. Posai V, Suksatan W, Choompunuch B, Koontalay A, Ounprasertsuk J, Sadang JM. Assessment of the health-promoting behaviors of hospitalized patients with non-communicable diseases

None. 
during the second wave of COVID-19. J Multidiscip Healthc 2021;14:2185-94.

13. Abel T, McQueen D. Critical health literacy and the COVID-19 crisis. Health Promot Int 2020;35:1612-3.

14. Huang CH, Talley PC, Lin CW, Huang RY, Liu IT, Chiang IH, et al. Factors associated with low health literacy among community-dwelling women in Taiwan. Women Health 2020;60:487501.

15. Wang J, Zhang R. COVID-19 in rural China: features, challenges and implications for the healthcare system.J Multidiscip Healthc 2021;14:1045-51.

16. Abdel-Latif M. The enigma of health literacy and COVID-19 pandemic. Public Health 2020;185:95-6.

17. Okan O, Bollweg TM, Berens EM, Hurrelmann K, Bauer U, Schaeffer D. Coronavirus-related health literacy: a cross-sectional study in adults during the COVID-19 infodemic in Germany. Int J Environ Res Public Health 2020;17:5503.

18. Lee MK, Oh J. Health-related quality of life in older adults: its association with health literacy, self-efficacy, social support, and health-promoting behavior. Healthcare (Basel) 2020;8:407.

19. Zhang F, Or PP, Chung JW. The effects of health literacy in influenza vaccination competencies among community-dwelling older adults in Hong Kong. BMC Geriatr 2020;20:103.

20. Yodmai K, Somrongthong R, Nanthamongkolchai S, Suksatan W. Effects of the Older Family Network Program on improving quality of life among older adults in Thailand. J Multidiscip Healthc 2021;14:1373-83.

21. Tran TV, Nguyen HC, Pham LV, Nguyen MH, Nguyen HC, Ha $\mathrm{TH}$, et al. Impacts and interactions of COVID-19 response involvement, health-related behaviours, health literacy on anxiety, depression and health-related quality of life among healthcare workers: a cross-sectional study. BMJ Open 2020;10:e041394.

22. Paakkari L, Okan O. COVID-19: health literacy is an underestimated problem. Lancet Public Health 2020;5:e249-50.

23. Patil U, Kostareva U, Hadley M, Manganello JA, Okan O, Dadaczynski K, et al. Health literacy, digital health literacy, and COVID-19 pandemic attitudes and behaviors in U.S. college students: implications for interventions. Int J Environ Res Public Health 2021; 18:3301.

24. Green LW, Kreuter MW. The PRECEDE-PROCEED model. In: Health promotion planning: an educational and ecological approach. 3rd ed. Boston, MA: McGraw-Hill; 1999. p. 32-43.

25. Faul F, Erdfelder E, Lang AG, Buchner A. G*Power 3: a flexible statistical power analysis program for the social, behavioral, and biomedical sciences. Behav Res Methods 2007;39:175-91.

26. Cobb S. Presidential Address-1976. Social support as a moderator of life stress. Psychosom Med 1976;38:300-14.
27. Ministry of Public Health. Health literacy. Nonthaburi, Thailand: Ministry of Public Health, Health Education Division; 2017.

28. Gautam V, S D, Rustagi N, Mittal A, Patel M, Shafi S, et al. Health literacy, preventive COVID 19 behaviour and adherence to chronic disease treatment during lockdown among patients registered at primary health facility in urban Jodhpur, Rajasthan. Diabetes Metab Syndr 2021;15:205-11.

29. Mackenbach JP. The persistence of health inequalities in modern welfare states: the explanation of a paradox. Soc Sci Med 2012; 75:761-9.

30. Panagioti M, Skevington SM, Hann M, Howells K, Blakemore A, Reeves D, et al. Effect of health literacy on the quality of life of older patients with long-term conditions: a large cohort study in UK general practice. Qual Life Res 2018;27:1257-68.

31. Wernly B, Wernly S, Magnano A, Paul E. Cardiovascular health care and health literacy among immigrants in Europe: a review of challenges and opportunities during the COVID-19 pandemic. Z Gesundh Wiss 2020 Oct 27 [Epub]. https://doi.org/10. 1007/s10389-020-01405-w.

32. Van den Broucke S. Why health promotion matters to the COVID-19 pandemic, and vice versa. Health Promot Int 2020; 35:181-6.

33. Kim J, Lee HY, Christensen MC, Merighi JR. Technology access and use, and their associations with social engagement among older adults: do women and men differ? J Gerontol B Psychol Sci Soc Sci 2017;72:836-45.

34. Liu Y, Meng H, Tu N, Liu D. The relationship between health literacy, social support, depression, and frailty among community-dwelling older patients with hypertension and diabetes in China. Front Public Health 2020;8:280.

35. Li Y, Hu L, Mao X, Shen Y, Xue H, Hou P, et al. Health literacy, social support, and care ability for caregivers of dementia patients: structural equation modeling. Geriatr Nurs 2020;41:6007.

36. Chen X, Li M, Kreps GL. Acculturation and health literacy among Chinese speakers in the USA with limited english proficiency. J Racial Ethn Health Disparities 2021;Feb 9; [Epub]. https://doi.org/10.1007/s40615-021-00979-9.

37. Wu F, Sheng Y. Social support network, social support, self-efficacy, health-promoting behavior and healthy aging among older adults: a pathway analysis. Arch Gerontol Geriatr 2019;85: 103934.

38. Widodo A, Prabandari YS, Sudiyanto A, Rahmat I. Increasing the quality of life of post-shackling patients through multilevel health promotion of shackling prevention. Bali Med J 2019;8:587-94.

39. Uemura K, Yamada M, Okamoto H. The effectiveness of an ac- 
tive learning program in promoting a healthy lifestyle among older adults with low health literacy: a randomized controlled trial. Gerontology 2021;67:25-35.

40. Yang Y, Zhang B, Meng H, Liu D, Sun M. Mediating effect of social support on the associations between health literacy, productive aging, and self-rated health among elderly Chinese adults in a newly urbanized community. Medicine (Baltimore) 2019; 98:e15162.

41. Laohathai S. Outcome comparison between video assisted thoracoscopic surgery and open thoracotomy on primary spontaneous pneumothorax in a tertiary hospital in Thailand. J Med Assoc Thailand 2021;104:100-4.

42. Suwanbamrung C, Le CN, Maneerattanasak S, Satian P, Talunkphet C, Nuprasert Y, et al. Developing and using a dengue pa- tient care guideline for patients admitted from households to primary care units and the district hospital: a community participatory approach in Southern Thailand. One Health 2020; 10:100168.

43. Jansen T, Rademakers J, Waverijn G, Verheij R, Osborne R, Heijmans $\mathrm{M}$. The role of health literacy in explaining the association between educational attainment and the use of out-of-hours primary care services in chronically ill people: a survey study. BMC Health Serv Res 2018;18:394.

44. van der Heide I, Wang J, Droomers M, Spreeuwenberg P, Rademakers J, Uiters E. The relationship between health, education, and health literacy: results from the Dutch Adult Literacy and Life Skills Survey.J Health Commun 2013;18(Suppl 1):172-84. 